\title{
Post-fabrication modification of electrospun nanofiber mats with polymer coating for membrane distillation applications
}

\author{
Journal of Membrane Science \\ Revised: January 18, 2017
}

Evyatar Shaulsky $^{\mathrm{a} \dagger}$, Siamak Nejati ${ }^{\mathrm{a}, \mathrm{b} \dagger}$, Chanhee Boo ${ }^{\mathrm{a}}$, François Perreault ${ }^{\mathrm{a}, \mathrm{c}}$, Chinedum O. Osuji ${ }^{\mathrm{a}}$, and Menachem Elimelech ${ }^{\mathrm{a}, *}$

${ }^{a}$ Department of Chemical and Environmental Engineering, Yale University,

New Haven, CT 06520-8286, USA

${ }^{b}$ Department of Chemical and Biomolecular Engineering, University of NebraskaLincoln, NE 68588-8286, USA

${ }^{c}$ School of Sustainable Engineering and the Built Environment, Arizona State University, Tempe, AZ 85287-3005, USA

* Corresponding author: Menachem Elimelech, Email: menachem.elimelech@yale.edu, Phone: (203) 432-2789.

${ }^{\dagger}$ E.S. and S.N. contributed equally to this work. 


\section{Abstract}

2 Post-treatment of electrospun nanofibers is a versatile and scalable approach for the fabrication

3 of membranes with controlled pore size, porosity, and morphology. In this study, we demonstrate

4 a novel solution-based approach for the fabrication of membrane distillation (MD) membranes

5 with adjustable pore size and performance through non-solvent induced phase separation of a

6 polymeric solution over an electrospun fiber mat. Poly(vinylidene fluoride-hexafluoropropylene)

7 (PVDF-HFP) was dissolved in a blend of acetone and dimethylacetamide and used to produce a

8 highly porous electrospun fiber mat with an average pore diameter of $\sim 1.2 \mu \mathrm{m}$. Surface coating

9 of the PVDF-HFP nanofibers with polyvinylidene fluoride (PVDF) through phase separation

10 enabled control of the membrane pore size by filling the empty domains between the fibers. The

11 coated fiber mats were characterized for their surface hydrophobicity, porosity, and structure.

12 The PVDF polymeric coating layer integrated within the electrospun mat decreased the average

13 pore diameter to $<0.6 \mu \mathrm{m}$ without compromising the surface hydrophobicity. By controlling the

14 depth of the PVDF coating layer within the substrate, we were able to fabricate robust

15 membranes with near complete salt rejection (>99.9\%) and a water flux of $30 \mathrm{~L} \mathrm{~m}^{-2} \mathrm{~h}^{-1}$ in direct

16 contact MD experiments with $40{ }^{\circ} \mathrm{C}$ temperature difference between the feed and permeate

17 solutions. This coating procedure is compatible with current roll-to-roll membrane fabrication

18 processes, making it a viable approach for large-scale fabrication of electrospun membranes with

19 exceptional performance for MD applications.

20 Keywords: Membrane distillation; Electrospun fiber mats; Asymmetric membrane; Microporous

21 membranes for MD; Adjustable pore size 


\section{Introduction}

Membrane distillation (MD) is an emerging separation process with potential applications for desalination of high salinity source waters [1,2]. MD can also be integrated with other processes to convert low-grade heat to electric power [3, 4] or for treatment of complex wastewaters [5-9]. Unlike conventional thermal desalination processes, which require high operating temperatures, MD can use a small temperature difference between a hot feed and a cold permeate to achieve a vapor pressure driving force across the membrane [10, 11]. The vapor pressure of water is weakly dependent on the feed water salinity [12], allowing MD to desalinate hypersaline source water that cannot be treated by pressure-driven membrane processes, such as reverse osmosis 33 (RO).

To achieve optimal performance, MD membranes should have two key properties: (i) high porosity to maximize water vapor flux and (ii) high hydrophobicity to avoid membrane pore wetting. Fabrication of high-performance MD membranes that meet these criteria via scalable and low-cost manufacturing techniques is a major technical challenge for the commercialization of MD technology, as current MD membranes still suffer from low permeate water flux and frequent pore wetting due to low hydrophobicity [13]. While novel membrane designs can overcome these limitations, such membranes must be fabricated using a scalable, environmentally benign, and low-cost technique.

Electrospun polymer nanofibers exhibit excellent characteristics suitable for MD applications, such as high surface roughness, high porosity, and mechanical robustness [14]. The ability to fabricate fibers with different morphology using various polymers renders this technology promising for a wide range of applications, from electronic devices to membrane filters and biomedical materials [15-19]. Previous studies have shown the feasibility of fabricating membranes, suitable for use in MD, using electrospinning of hydrophobic polymers, including polyvinylidene fluoride (PVDF) and poly(vinylidene fluoride-co-hexafluoropropylene) (PVDF-HFP) [20, 21].

PVDF-HFP exhibits low surface energy [22, 23], which makes it an attractive material for the fabrication of MD membranes with improved hydrophobicity [24]. Additionally, electrospun

52 nanofiber mats exhibit high surface roughness, which results in an increased air gap at the 53 surface interface, and thus enhances surface hydrophobicity. These promising features render 
54 electrospun fiber mats prepared using PVDF-HFP a suitable platform to fabricate high55 performance MD membrane.

56 Due to the random assembly of fibers and the lack of chemical bonding between them, fine 57 control of the membrane structure, including pore size and pore size distribution, is not 58 achievable by electrospinning [24]. To address these limitations, electrospun mats were treated 59 post-fabrication using hot pressing [20,25], which fuses the fibers on the top layer together and 60 reduces the overall membrane porosity and surface roughness. However, the latter compromises 61 the surface hydrophobicity, thereby increasing the wetting propensity of the membrane. 62 Alternative strategies that allow fine-tuning of the porosity and structural integrity of nanofiber 63 mats would thus represent a significant step toward the development of more efficient 64 electrospun nanofiber MD membranes.

65 In this study, we developed a simple and scalable method to fabricate robust MD membranes 66 based on electrospun nanofibers. We prepared an electrospun PVDF-HFP fiber mat substrate and 67 then applied a PVDF coating on the fibers using a simple phase inversion approach. The 68 additional polymer coating not only decreases the pore size and the porosity but also links the 69 fibers, which substantially improves the mechanical stability of the fiber mat. The fabricated 70 membranes demonstrated a relatively high water flux and nearly complete salt rejection in a 71 direct contact membrane distillation (DCMD) test. Our results highlight the potential of this 72 method for scalable production of membranes for membrane distillation applications.

\section{2. Materials and methods}

\section{$75 \quad$ 2.1. Materials and chemicals}

Poly(vinylidene fluoride-co-hexafluoropropylene) (PVDF-HFP) (MW: 455 kDa), 77 polyvinylidene fluoride (PVDF) (MW: $275 \mathrm{kDa}), N, N$-Dimethylacetamide (DMAc) (99.5\%), and 78 triethyl phosphate (TEP) (99.8\%) were purchased from Sigma-Aldrich and used as received. 79 Acetone (99.5\%) and 2-propanol (99.5\%) were acquired from J. T. Baker (Phillipsburg, NJ). 80 Ultrapure water was obtained from a Millipore Milli-Q system.

\subsection{PVDF-HFP substrate fabrication}


$1 / 10(\mathrm{w} / \mathrm{v})$ PVDF-HFP in a 4/6 (v/v) mixture of DMAc and acetone was prepared by

83 dissolving the polymer in the solvent at room temperature under overnight stirring. A $10 \mathrm{~mL}$

84 PVDF-HFP solution was loaded in a Luer-Lok Tip syringe (Becton Dickinson \& Co., Franklin

85 Lakes, NJ), to which an $18 \mathrm{G} \times 1$ inch precision glide needle (Becton Dickinson \& Co.) was

86 attached. The syringe was placed horizontally on the syringe pump (Harvard Apparatus,

87 Plymouth Meeting, PA), and an electrode of high-voltage supply (Gamma High Voltage

88 Research Inc., Ormond Beach, FL) was clamped to the needle tip. The needle to collector

89 distance was kept at $15 \mathrm{~cm}$. A $10.5 \mathrm{~cm} \times 38.5 \mathrm{~cm}$ aluminum foil was installed on a rotating drum

90 which moved at a speed of $\sim 16 \mathrm{~cm} / \mathrm{min}$ to collect the fibers. The flow rate and the applied

91 voltage were set to $1 \mathrm{~mL} / \mathrm{h}$ and $18.5 \mathrm{kV}$, respectively. The electrospinning was performed in a

92 closed chamber under controlled temperature $\left(30{ }^{\circ} \mathrm{C}\right)$ and relative humidity $(45-50 \%)$. Fibers

93 were collected for 10 hours, and the collected fiber mat was dried in an oven at $60{ }^{\circ} \mathrm{C}$ for 12

94 hours.

\section{$95 \quad 2.3$ PVDF coating of the substrate}

96 A $15 \%$ (w/v) PVDF solution was prepared by mixing a known amount of polymer in TEP at

$97 \quad 70{ }^{\circ} \mathrm{C}$ and continuous stirring for 8 hours. The solution was cooled to room temperature before

98 use. The prepared PVDF solution was loaded on an aluminum rod by rolling it on a glass plate

99 covered with the polymer solution. Then, this PVDF-impregnated rod was rolled on the PVDF-

100 HFP electrospun substrate to achieve a thin layer PVDF coating. Immediately after PVDF

101 coating, the substrate was submerged in a 2-propanol coagulation bath to induce phase inversion

102 of the PVDF, as illustrated in Fig. 1. For the thick PVDF layer coating, the PVDF solution was

103 spread on the PVDF-HFP electrospun substrate using a doctor blade casting knife and allowed to

104 penetrate all the way through the fiber mat. PVDF-coated PVDF-HFP electrospun substrates on a

105 glass plate were left for 20 minutes in the coagulation bath to induce phase inversion, and then

106 transferred to a water bath where they were stored overnight to remove the residual solvent.

107 After solvent removal, the membranes were dried in an oven at $60{ }^{\circ} \mathrm{C}$ for 24 hours. 


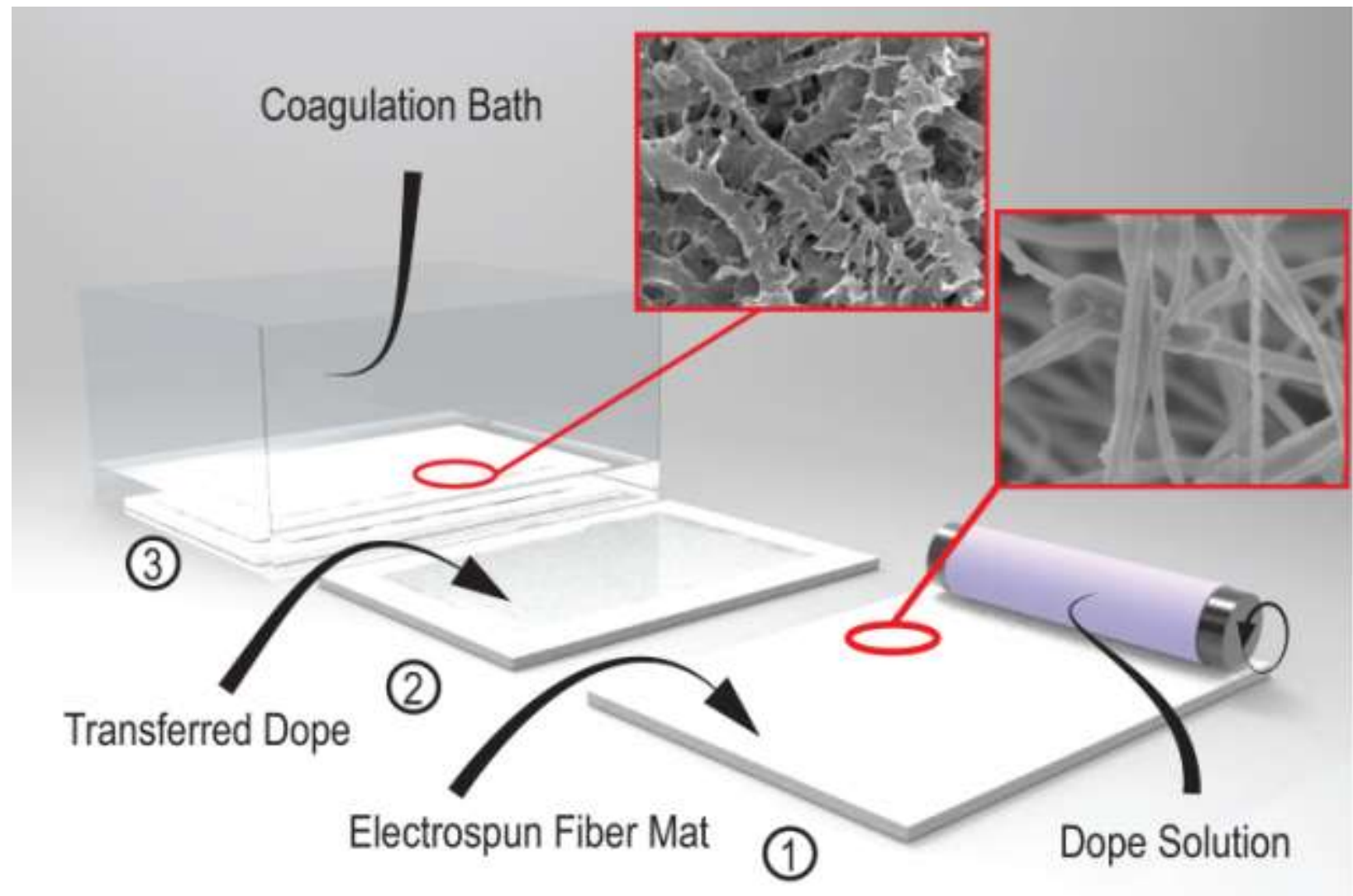

110 Fig. 1. Schematic of the process for controlled infiltration of the PVDF dope solution (i.e., PVDF 111 dissolved in TEP) within the PVDF-HFP electrospun nanofiber mat and thin layer coating of the 112 nanofiber mats by non-solvent induced phase separation. Step 1: Thin layer of PVDF solution is 113 loaded onto aluminum rod. Step 2: The PVDF solution layer is transferred to the fiber mat. Step 114 3: The fiber mat coated with dope solution is submerged in a coagulation bath for phase 115 separation of the PVDF coating layer.

\subsection{Membrane characterization}

2.4.1 X-ray photoelectron spectroscopy (XPS)

XPS was performed on a Physical Electronics instrument (PHI 5000, VersaProbe) with a 120 scanning monochromatic source from an $\mathrm{Al}$ anode with dual beam charge neutralization. Both 121 survey and high-resolution spectra were acquired over a spot of approximately $200 \mu \mathrm{m}$ in 
122 diameter with $\sim 50 \mathrm{~W}$ beam power. High-resolution XPS spectra of C1s were acquired with 0.1

$123 \mathrm{eV}$ resolution at $23.5 \mathrm{eV}$ pass energy with $200 \mathrm{~ms}$ dwell time, and averaged over 10 scans.

$124 \quad$ 2.4.2 Scanning electron microscopy

125 All micrographs were acquired using a Hitachi Ultra-High-Resolution Analytical Field 126 Emission Scanning Electron Microscope (FE-SEM) SU-70. To obtain cross-section images, 127 membranes were dried at room temperature and freeze-fractured using liquid nitrogen before 128 mounting on the sample holder. A Denton chromium sputtering machine equipped with a quartz 129 crystal sensor and film deposition monitor (INFICON) was used to coat the sample surface with 130 a $15 \mathrm{~nm}$ thick chromium layer. The coated samples were immediately imaged to avoid oxidation 131 of the conductive coating.

\section{$132 \quad$ 2.4.3 Transmission electron microscopy (TEM)}

133 For TEM imaging, nanofibers were immersed in epoxy resin (EMbed 812, Electron 134 Microscopy Sciences, Hatfield, PA) and deaerated for 4 hours at room temperature under 135 vacuum. The samples were cured at $58{ }^{\circ} \mathrm{C}$ for 48 hours. Embedded samples were cut into 0.07 $136 \mu \mathrm{m}$ slices and placed on a copper grid (Holey Carbon, 300 mesh, Electron Microscopy Sciences, 137 Hatfield, PA). Samples were visualized with an FEI Tecnai Osiris microscope (FEI, Hillsboro, 138 OR), operating at an acceleration voltage of $200 \mathrm{kV}$. Elemental mapping was performed by 139 solid-angle X-ray spectroscopy.

\subsubsection{Contact angle}

141 Water contact angles for the top and bottom sides of the membranes were measured by an 142 optical tensiometer (OneAttension, Biolin scientific instrument) using the sessile drop method. A $1433-\mu \mathrm{L}$ DI water droplet was placed on the air-dried membrane sample and photographed using a 144 digital camera. The left and right contact angles were determined from digital images with post145 processing software (OneAttension software). Measurement for each data point was conducted at 146 three different locations on the same membrane coupon and averaged.

\subsubsection{Pore size distribution}

148 The pore size distribution was evaluated by the wet/dry flow method [26] using a custom149 made porometer setup (Fig. S1), which uses a small gas-filtration unit with a stainless steel filter 150 holder (KS 25A, Advantec MFS) having an effective area of $\sim 3.9 \mathrm{~cm}^{2}$ (50\% open). The 
151 membrane was cut to fit the holder size. A commercial wetting liquid (Porefil), having a low 152 surface tension $(16 \mathrm{mN} / \mathrm{m})$ and vapor pressure (399 $\mathrm{Pa}$ at $298 \mathrm{~K})$, was used to wet the membrane.

153 A digital pressure regulator (Control Air Inc., 900-CHA) was used to control the nitrogen gas

154 pressure, and gas flow and pressure across the membrane were recorded by a pressure transducer

155 (Honeywell, px2an1xx100psaax) and a flow meter (Porter MPC20).

\subsubsection{Porosity}

157 Film porosity was estimated by a gravimetric method. The oven-dried membrane was 158 weighed and subsequently submerged in a container filled with 2-propanol for one hour. The wet 159 membrane was taken out of the 2-propanol, sandwiched between nylon fabrics (VWR), and

160 further padded with paper wipes to remove the excess 2-propanol from the surface. Wet sample 161 weight was monitored using an analytical balance (Denver PI-214A). The weight decrease was 162 recorded to a laptop in one-second intervals until the sample was fully dried, and no changes in 163 weight were observed. The porosity $(\varepsilon)$ was estimated using the equation:

164

$$
\varepsilon=\frac{V_{\text {pore }}}{V_{\text {total }}}=\frac{\frac{m_{I P A}}{\rho_{I P A}}}{V_{\text {pore }}+V_{\text {Polymer }}}=\frac{\frac{m_{I P A}}{\rho_{I P A}}}{\frac{m_{I P A}}{\rho_{I P A}}+\frac{m_{\text {Polymer }}}{\rho_{\text {Polymer }}}}
$$

165 where $V, m$, and $\rho$ are the volume, mass, and density, respectively, and IPA indicates the 2166 propanol. The test was performed three times, and the porosity values were averaged.

\section{2.4.7 Liquid Entry Pressure}

168 The liquid entry pressure (LEP) was measured using the porometer previously described. DI 169 water was placed on the membrane and the pressure in the sample holder was increased by 170 flowing nitrogen gas. As the applied pressure increases, the gas pushes the water out of the 171 membrane pores. The pressure at which the gas flow is detected by a flow meter was reported as 172 the liquid entry pressure.

1732.4 .8 tensile test

174 We evaluated the mechanical properties of the electrospun PVDF-HFP membranes using a 175 tensile tester (Series 5542, Instron, MA). A membrane sample $(1 \mathrm{~cm} \times 3 \mathrm{~cm})$ was vertically 
176 mounted on the tester using two grippers at its ends, leaving a $2 \mathrm{~cm}$-gauge length for mechanical

177 loading. Load-deformation data were recorded at a rate of $0.5 \mathrm{~mm} / \mathrm{sec}$.

178

179

180

181

182

183

\subsection{Evaluation of Membrane Performance}

The performance of fabricated membranes was evaluated using a laboratory-scale direct contact membrane distillation (DCMD) unit (Fig. S2). The membranes were inserted into the custom-built acryl cell with an effective membrane area of $20.02 \mathrm{~cm}^{2}$ for both distillate (permeate) and feed sides. Hot feed and cold distillate streams were circulated using two variable gear pumps (Cole-Parmer, Vernon Hills, IL) and the temperatures were kept constant at 60 and $20{ }^{\circ} \mathrm{C}$, respectively, using two recirculating water baths (Polystat Standard, Cole-Parmer, Vernon Hills, IL) [27]. Spacers were inserted in both feed and distillate channels to support and maintain the membrane geometry in the cell. The applied feed cross flow velocity was two times higher than permeate stream (i.e., $15.2 \mathrm{~cm} / \mathrm{s}$ for feed versus $7.6 \mathrm{~cm} / \mathrm{s}$ for permeate) to facilitate the detection of membrane wetting by imposing a slightly higher hydraulic pressure on the feed side. Water vapor flux, $J_{w}$, across the membrane was measured by monitoring the increase in distillate mass using a digital balance (CUW 6200H, CAS). The distillate weight gain was recorded in a laptop at one-minute intervals. Salt passage was monitored by measuring the salt concentration in distillate using a calibrated conductivity meter (Oakton Instruments, Vernon Hills, IL) at oneminute intervals.

\section{Results and discussion}

\subsection{Membrane Structure}

The control substrate was prepared by electrospinning a solution of PVDF-HFP in a mixture of DMAc and acetone [28]. An electrospun fiber mat composed of nanofibers with an average diameter of $477 \mathrm{~nm}$ and an overall thickness of $70 \mu \mathrm{m}$ was collected on an aluminum foil. We gained control of the effective pore diameter of the fabricated membranes by controlling the infiltration depth of a PVDF solution within the fiber mat, followed by solidifying the polymer by phase inversion. TEP (i.e., the solvent used for PVDF coating solution) does not dissolve PVDF-HFP at room temperature, which enables PVDF coating on the PVDF-HFP electrospun fibers without damaging the fibers. 
Two different polymer coating approaches were used to control the penetration depth of the 206 dope solution within the substrate and, consequently, the thickness of the polymer (PVDF) film 207 deposited within the fiber mat. In the first approach, a PVDF solution in TEP was spread evenly 208 on the PVDF-HFP fiber mats using a knife casting blade, which resulted in a deep penetration of 209 the polymer solution inside the fiber mat. For the second approach, the amount of PVDF applied 210 to the fiber mat was controlled by wetting a rolling rod uniformly with a known amount of 211 polymer solution. By rolling the rod against the fiber mat, the solution was transferred to the 212 substrate. The high porosity of the substrate, combined with the low surface tension of the PVDF 213 in TEP solution (38.2 and $\sim 36.5 \mathrm{mN} / \mathrm{m}$ for TEP and PVDF, respectively [29]), results in 214 effective permeation of the polymer solution into the substrate pores by capillary action [30].

215 The PVDF-coated fiber mat was subsequently submerged in the coagulation bath to 216 precipitate PVDF on the fibers via phase separation. During the phase separation process, the 217 solid polymer structure collapses within the substrate voids. As a result, most of the polymer 218 coats the nanofibers and less remains within the voids between fibers.

219 The change in membrane morphology after PVDF coating is visualized by scanning electron 220 microscopy (SEM). Figs. 2A and 2B show that the penetration depth of the polymer solution 221 within the electrospun mat was significantly different for the two different coating approaches, as 222 indicated by the depth of the solid PVDF residual, visible within the fiber mat. The control over 223 the penetration depth of dope solution within the substrate provides a simple route to manipulate 224 the membrane structure. The ease of processing can make this method a viable approach to 225 fabricating membranes in a roll-to-roll fashion [31]. 
Thin Layer Casting

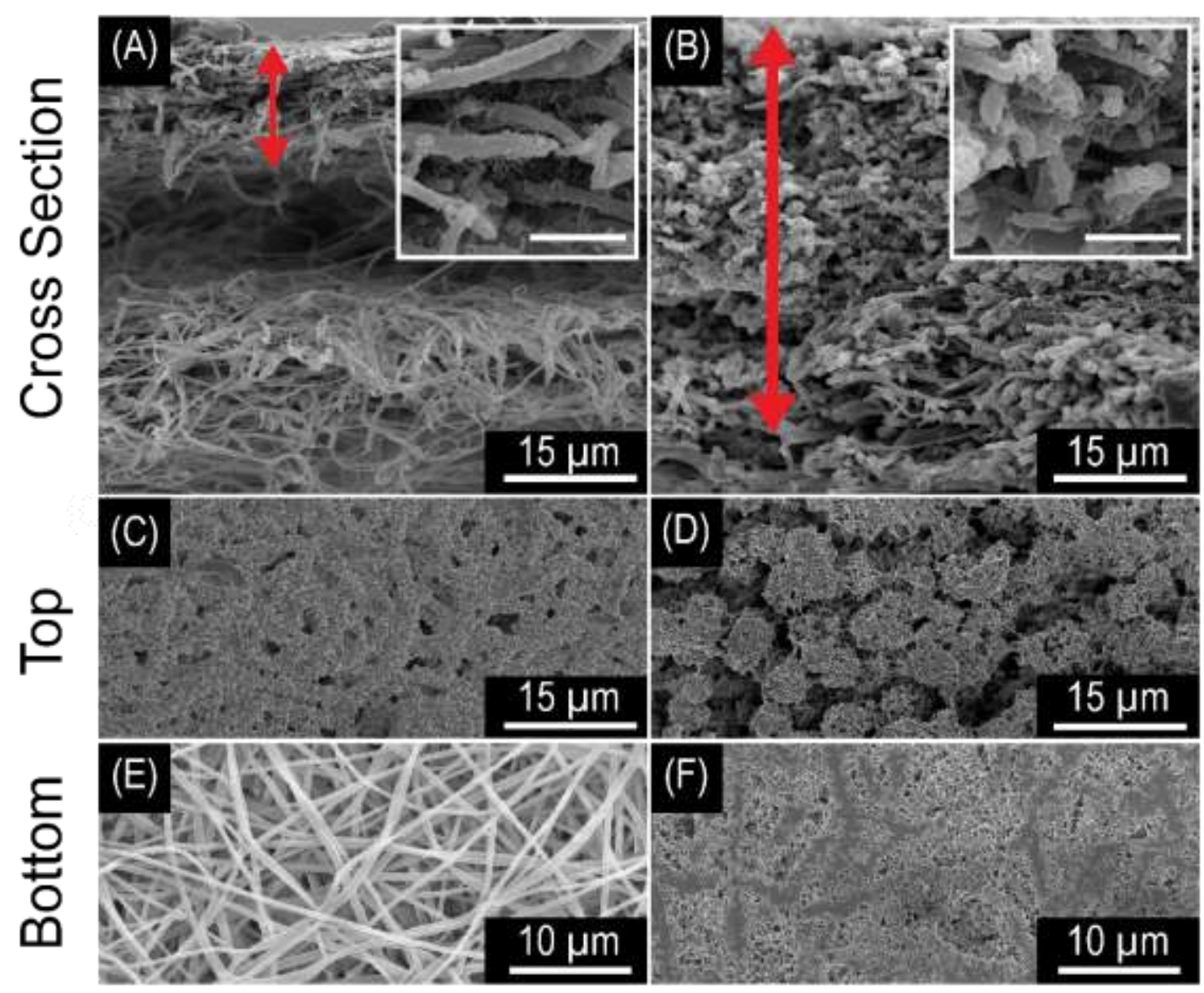

228 Fig. 2. Morphology of the PVDF-HFP electrospun fiber mats after coating with PVDF via a thin

229 layer casting method and a knife casting method. The SEM micrographs (A) and (B) are cross 230 sections, (C) and (D) are top surfaces, and (E) and (F) are bottom surfaces of the modified 231 PVDF-HFP electrospun fiber mats. The red arrows in parts (A) and (B) indicate penetration 232 depth of the PVDF coating solution. The insets in parts (A) and (B) are high-magnification 233 images and scale bars indicate $1 \mu \mathrm{m}$.

234 Due to a low solubility parameter of the PVDF-2-propanol (nonsolvent coagulation medium) 235 pair (i.e., $9.7 \mathrm{MPa}^{0.5}$ ), the liquid-liquid de-mixing is delayed and the formation of a dense skin 236 layer is prevented [32-34]. As shown in Fig. 2C, when the dope solution penetration depth was 237 limited to $10-20 \mu \mathrm{m}$ from the top of the mat, a relatively homogeneous PVDF coating around the 238 fibers was formed upon phase inversion. In contrast, when the knife casting method was used, 
239 crystalline domains, visible as micron-sized particles (Fig. 2D), were formed on the top. These 240 crystalline domains are attributed to the phase inversion of PVDF by the soft coagulation 241 medium (i.e., 2-propanol) as shown in previous studies [35, 36].

242 The bottom surface of the mat was also visualized to verify the penetration depth of the 243 PVDF solution. When the dope solution penetration is controlled, the morphology of the fiber on 244 the bottom side of the electrospun mat remains intact, demonstrating a thin layer PVDF coating 245 within a confined thin layer of the PVDF-HFP substrate (Fig. 2E). On the other hand, when 246 PVDF is spread on the fiber mat using a casting knife, the polymer solution penetrates deep into 247 the mat. Consequently, as shown in Fig. 2F, the bottom side of the membrane contains smooth 248 patches that are indicative of PVDF phase inversion at the interface between a glass plate and the 249 substrate surface. As shown in the cross-sectional SEM (Fig. 2A), when the penetration depth of 250 the polymer solution is confined within the porous substrate, the final membrane structure is 251 asymmetric. The asymmetric membrane structure is crucial for MD performance, as discussed 252 later in Section $3.5[34,37]$.

253 To further confirm the PVDF coating on the PVDF-HFP electrospun fibers, we visualized the 254 cross section of the fibers by TEM imaging. The nanofibers were embedded in an epoxy resin 255 and microtomed into $70 \mathrm{~nm}$ thin-slices. An additional PVDF layer was observed around the 256 fibers upon non-solvent induced phase inversion (Fig. 3). Images also indicate that polymer 257 residues occupy the space between individual fibers and create a stable linkage between them, 258 which can be easily observed when the fibers are closer to each other (Figs. 3B and 3D). This 259 PVDF bridging between fibers substantially improves the mechanical stability of the mat, as we 260 discuss later. 


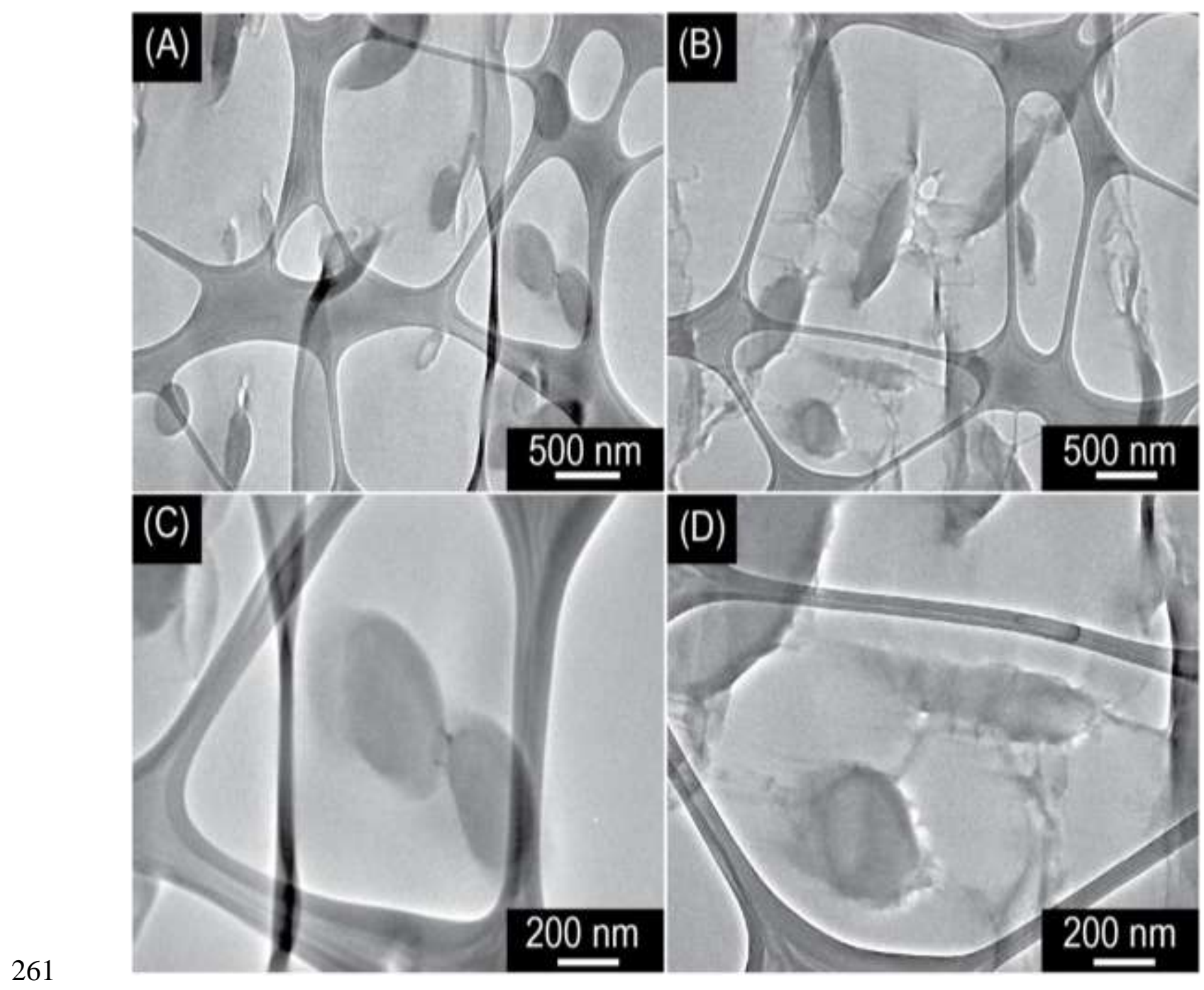

263 Fig. 3. Transmission electron micrographs of the fiber cross-section showing the coated and 264 uncoated fiber morphology. Electrospun PVDF-HFP fibers (A, C) show a well-defined round 265 morphology while PVDF-coated fibers have a polymeric web-like structure bridging the fibers 266 (B, D).

\subsection{Membrane porosity and pore size distribution}

The pore size distribution was evaluated by a wet/dry flow method with perfluoroether 269 (Porefil, Porometer, Belgium) as a wetting liquid using a lab-made porometer (Fig. S1). Due to 270 the low surface tension $(16 \mathrm{mN} / \mathrm{m})$, Porefil can fully wet the membrane, such that the contact 271 angle between the membrane surface and the wetting liquid, $\theta$, is assumed to be zero. The 272 membrane pore diameter, $d_{p}$, was calculated using the Laplace equation [26]: 
$273 \mathrm{~d}_{\mathrm{P}}=\frac{4 \sigma}{\Delta P} \cos \theta$

274 where $\Delta P$ is the pressure difference across the membrane and $\sigma$ is the surface tension of the 275 wetting liquid. The additional polymer coating around the fibers was found to decrease the 276 effective pore size of the membrane, as observed by a shift of the pore diameter distribution 277 towards smaller values (Fig. 4). Also, the deeper penetration of the PVDF solution into the fiber 278 mat resulted in a narrower pore size distribution. The results suggest that the effective pore size 279 distribution can be tuned by adjusting the penetration depth of the polymer coating solution. To 280 enhance the versatility of this technique and gain better control over the pore size, other phase 281 inversion parameters, including the viscosity of the dope solution and coagulation bath media, 282 can be further examined [38-40].

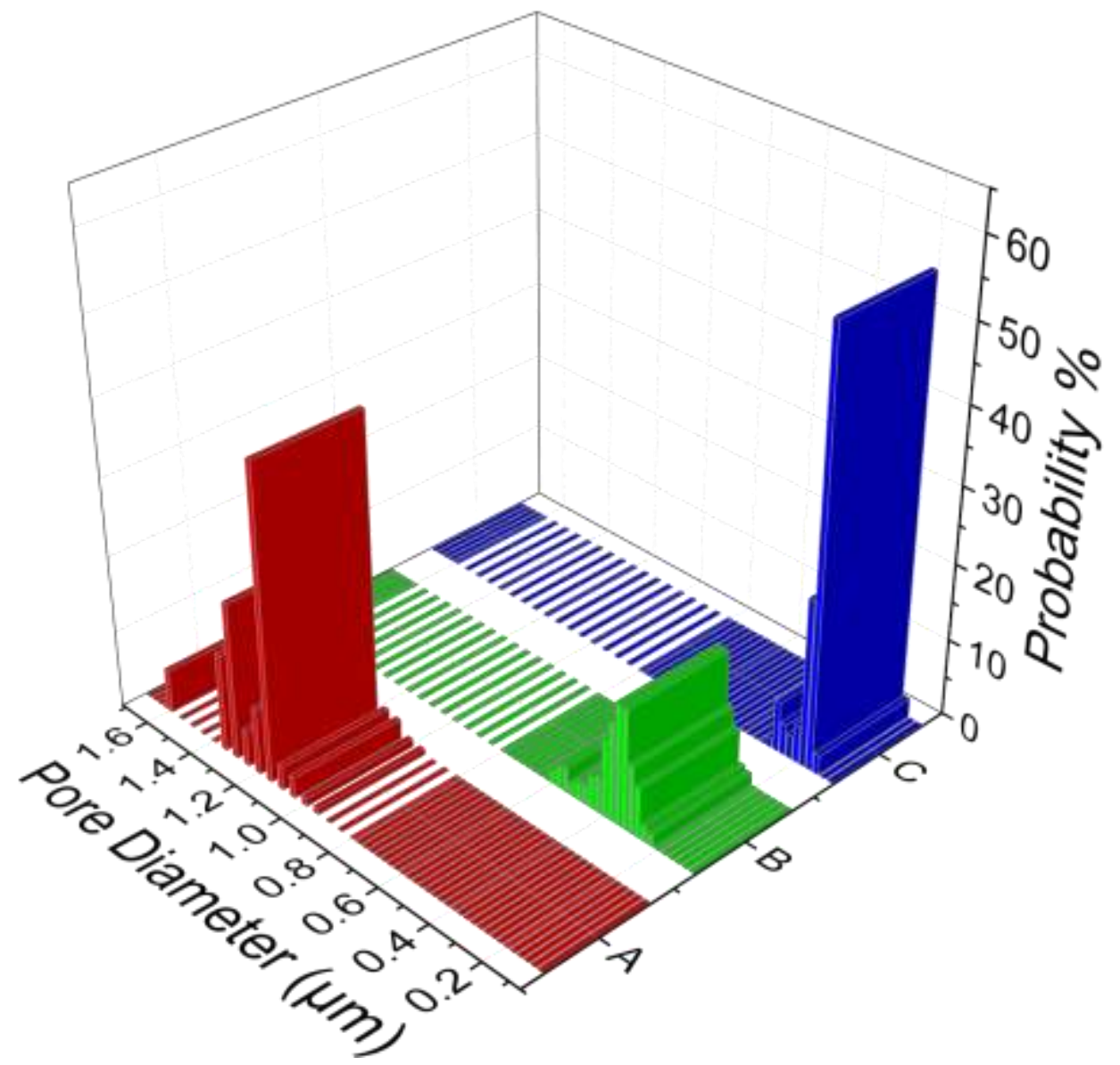

284 Fig. 4. Pore size distributions of (A) control PVDF-HFP electrospun substrate, (B) PVDF-HFP 285 substrate modified with a shallow penetration of PVDF dope solution, and (C) PVDF-HFP 
substrate modified with a deep penetration of PVDF dope solution. Pore size distribution was measured by a wet/dry capillary flow method using a custom-built porometer.

The porosities of the control and modified membranes are provided in Table 1. It is evident that the overall membrane porosity is reduced as the polymer loading within the fiber mat is increased $[41,42]$. This reduction in porosity is expected, because the void volume between the

292 fibers is occupied by the PVDF coating through phase separation. The decrease in porosity was 293 proportional to the thickness of the coating layer. The porosities of the control, thin layer casting, 294 and knife casting modified membranes were estimated to be 90.6, 80.2, and 70.2\%, respectively.

Table 1. Effect of infiltration depth of dope solution on the properties of the electrospun substrate.

\begin{tabular}{|c|c|c|c|c|c|c|c|}
\hline & \multicolumn{2}{|c|}{ Contact Angle $\left(^{\circ}\right.$ ) } & \multirow[b]{2}{*}{$\begin{array}{c}\text { Porosity, } \varepsilon \\
(\%)\end{array}$} & \multirow[b]{2}{*}{$\begin{array}{l}\text { Liquid Entry } \\
\text { Pressure } \\
\text { (kPa) }\end{array}$} & \multicolumn{2}{|c|}{ Tensile measurement } & \multirow{2}{*}{$\begin{array}{c}\text { Total } \\
\text { thickness } \\
\quad(\mu \mathrm{m})\end{array}$} \\
\hline & Top & Bottom & & & $\begin{array}{c}\text { Max. } \\
\text { Force (N) }\end{array}$ & $\begin{array}{l}\text { Max. } \\
\text { Strain } \\
(\mathrm{mm})\end{array}$ & \\
\hline Control & \multicolumn{2}{|c|}{$131.9 \pm 2.0$} & $90.6 \pm 0.6$ & 56 & $2.38 \pm 0.24$ & $16.9 \pm 3.28$ & 77 \\
\hline $\begin{array}{l}\text { Thin } \\
\text { layer }\end{array}$ & $\begin{array}{c}130.6 \pm \\
1.1\end{array}$ & $\begin{array}{c}132.1 \pm \\
1.7\end{array}$ & $80.2 \pm 0.3$ & 163 & $3.38 \pm 0.13$ & $24.2 \pm 1.41$ & 75 \\
\hline $\begin{array}{c}\text { Casting } \\
\text { knife }\end{array}$ & $\begin{array}{c}133.3 \pm \\
1.7\end{array}$ & $\begin{array}{c}126.9 \pm \\
2.4\end{array}$ & $70.2 \pm 0.2$ & 325 & $2.78 \pm 0.09$ & $2.1 \pm 1.34$ & 71 \\
\hline
\end{tabular}

\subsection{Membrane surface chemical and wetting properties}

X-ray photoelectron spectroscopy (XPS) analysis was performed on the control and modified PVDF-HFP electrospun fiber mats to elucidate the surface chemistry of the membrane before and after polymer coating. As shown in Fig. 5A, the C1s spectrum of the control PVDF-HFP nanofibers exhibits two major peaks attributable to the fluorinated carbon species and one peak ascribed to the hydrocarbon species. The peaks at 293.2 and $290.6 \mathrm{eV}$ are assigned to the $\mathrm{CF}_{3}$ and $\mathrm{CF}_{2}$ species, respectively, and the peak at $286.4 \mathrm{eV}$ is ascribed to the $\mathrm{CH}_{2}$ moiety of the PVDF block. The appearance of peaks at 284.6, 285.8, and $286.5 \mathrm{eV}$ in the $\mathrm{C} 1 \mathrm{~s}$ spectrum was attributed to adventitious carbon contamination present in the environment [43]. As shown in Fig. $5 \mathrm{~A}$, the concentration of $\mathrm{CF}_{3}$ species is significantly smaller compared to the $\mathrm{CF}_{2}$ species. Under 
308 humid electrospinning conditions, it was expected that the HFP block orientation would be 309 inward in order to minimize the surface energy. This inward orientation can explain the 310 significant mismatch between the stoichiometry ratio of the $\mathrm{CF}_{3}$ in PVDF-HFP and the measured $311 \mathrm{CF}_{3}$ concentration on the surface of the control sample.

312 Fig. 5B shows the C1s spectrum of the PVDF-coated PVDF-HFP electrospun fibers. Upon 313 coating of the fiber with $\mathrm{PVDF}$, the $\mathrm{CF}_{3}$ peak at $293.2 \mathrm{eV}$ completely vanished, and the most 314 prominent features of the spectrum were assigned to the PVDF layer. The minimal change in the 315 XPS spectrum of the sample after coating suggests that the PVDF coating has minor influence on 316 the chemical composition of the membrane surface.

(A) Control PVDF-HFP Substrate

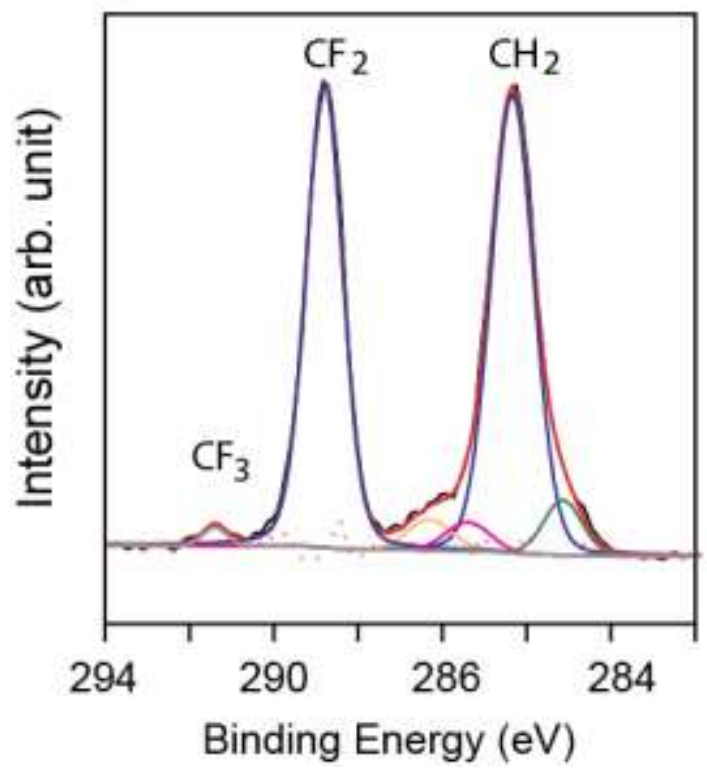

(B) Substrate with PVDF Coating

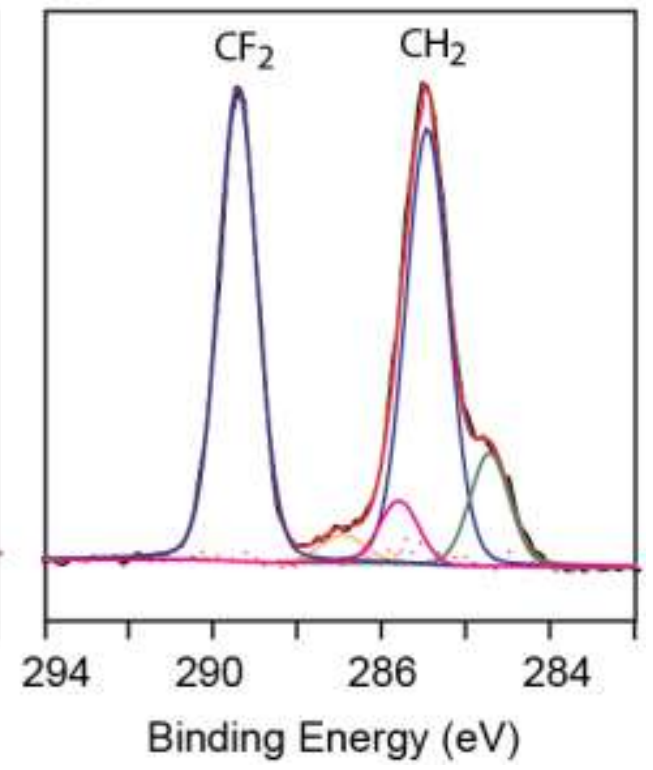

319 Fig. 5. X-ray photoelectron spectroscopy spectrum showing the C1s region of the (A) 320 electrospun fiber mat and (B) fiber mat after post processing. The unlabeled peaks are attributed 321 to the adventitious carbon contamination, which has an overlap with the range, in which the CF 322 binding energy range is present in PVDF-HFP.

As indicated earlier, surface hydrophobicity or wettability of the membrane is dependent on 325 its surface energy and surface roughness [44-46]. To evaluate surface hydrophobicity, we 326 measured the water contact angle of the membranes using the sessile drop method (Table 1) [47]. 
327 Interestingly, no significant difference was observed between the contact angles of the control 328 PVDF-HFP substrate $\left(131.9^{\circ}\right)$ and the PVDF-HFP fiber mat modified with a thin layer PVDF 329 coating $\left(130.6^{\circ}\right)$. This observation, along with the minimal difference in the chemical 330 composition (based on XPS analysis) of the PVDF-HFP electrospun fiber mats before and after 331 PVDF coating, suggests that membrane surface roughness has not been significantly influenced 332 by the thin layer PVDF coating.

333 The water contact angle on the top surface of the PVDF-HFP electrospun mat coated with a 334 thick PVDF layer by a knife casting method was $133.3^{\circ}$, higher than the control and the thin 335 layer modified PVDF-HFP electrospun fiber mats. For the membranes modified with a knife 336 casting technique, a globular crystalline polymer domain on the top layer (shown in Figure 2D) 337 is expected to introduce additional surface roughness, thereby resulting in enhanced surface

338 hydrophobicity. The water contact angle on the bottom side of this membrane was smaller than 339 the control PVDF-HFP substrate $\left(127^{\circ}\right.$ and $132^{\circ}$ for the thin layer modified and the control 340 PVDF-HFP electrospun mats, respectively). This difference can be explained by a smoother 341 surface morphology, shown in Figure 2 F, created by PVDF penetration and deposition at the 342 glass plate substrate and fiber interface.

\subsection{Membrane liquid entry pressure and mechanical properties}

345 We also measured the membrane liquid entry pressure for water (LEPw), defined as the minimum pressure required to break the water meniscus at the membrane pore opening [48],

347 which leads to pore wetting. The LEPw is influenced by the effective solid-liquid contact angle, 348 the membrane pore size, and the pore shape, as described by the Laplace equation [49, 50]. The 349 correlation between a rougher surface and higher contact angle is straightforward under the non350 wetting Cassie-Baxter state. An increase in surface roughness leads to higher contact angle and, 351 correspondingly, a higher LEP of a membranes with the same pore size [51, 52]. Because the 352 changes in membrane surface hydrophobicity were relatively small, as evidenced by previously 353 discussed contact angle measurements, the differences in LEPw can be explained by the change 354 in the pore size distribution of the membranes [26]. As expected, a decrease in the maximum 355 pore size leads to an increase in LEPw. The LEPw values for the control electrospun substrate 
and thin layer and knife casting modified membranes were 56, 163, and $325 \mathrm{kPa}$, respectively

357 (Table 1), which is in line with the observed shift in the pore size distribution (Fig. 4).

Tensile tests were performed to evaluate the effect of the PVDF coating on the mechanical properties of the membrane. Results from tensile tests are presented in Fig. S5 of the Supplementary Data and are also summarized in Table 1. We found that a thick coating layer achieved by a knife casting method increased the maximum tensile strength of the electrospun fiber mats by $16.8 \%$. However, this thick polymer coating inhibited the ability of the fibers to strain and thus decreased the maximum strain by $80.4 \%$. In contrast, thin layer PVDF coating linked fibers together without compromising the fiber flexibility, resulting in improvements of both maximum strain and strength of the membrane by 43.2 and $42 \%$, respectively.

\subsection{Membrane performance}

The performance of the control and modified membranes was evaluated using a laboratoryscale direct contact membrane distillation (DCMD) unit (Fig. S2). Hot feed and cold distillate streams were circulated at temperatures of 60 and $20^{\circ} \mathrm{C}$, respectively. Distillate flux, $J_{w}$, across the membrane was measured by monitoring the increase in distillate mass using a digital balance, and salt passage was monitored by the change in salt concentration of the distillate using an electric conductivity meter.

We performed DCMD experiments at a feed cross-flow velocity two times higher than the permeate stream (i.e., $15.2 \mathrm{~cm} / \mathrm{s}$ for feed versus $7.6 \mathrm{~cm} / \mathrm{s}$ for permeate) to facilitate membrane wetting within a relatively short testing time by imposing a slightly higher hydraulic pressure on the feed side. The control PVDF-HFP electrospun substrate produced very high permeate flux; however, the presence of relatively large pores caused rapid wetting of the membrane (Fig. 6A). The conductivity of the distillate for the control membrane started to increase exponentially 30 minutes after the onset of the DCMD experiment, clearly indicating wetting of the membrane.

On the other hand, the membranes with PVDF coating exhibited stable DCMD performance 383 fiber substrate with PVDF decreased the effective pore size (Fig. 4) without compromising 384 surface hydrophobicity (Table 1), thereby resulting in higher membrane wetting resistance. This 385 enhanced wetting resistance of the PVDF-coated electrospun substrates enabled a stable 
386 desalination performance under MD operation conditions that facilitate wetting (i.e., two times 387 higher feed cross flow velocity than distillate stream).

388 While the salt rejection has been significantly improved after PVDF coating, the decrease in 389 pore size (Fig. 4) and porosity (Table 1) following the post-fabrication modification negatively 390 impacted the membrane water vapor flux[53]. Comparing membrane flux performance (Fig. 6) 391 and membrane physical properties (Table 1) clearly showed that thicker PVDF coatings on the 392 PVDF-HFP substrates resulted in a lower water vapor flux. The highly porous ( 90\%) control 393 electrospun mats exhibited a high water flux of $\sim 70 \mathrm{~L} \mathrm{~m}^{-2} \mathrm{~h}^{-1}$, while membranes coated through a 394 thin layer and a knife casting methods showed lower water fluxes of $\sim 30$ and $\sim 10 \mathrm{~L} \mathrm{~m}^{-2} \mathrm{~h}^{-1}$, 395 respectively.

396 Although additional polymer coating reduced water vapor permeability, the membrane 397 modified with a thin layer coating still exhibited a fairly high water flux of $\sim 30 \mathrm{~L} \mathrm{~m}^{-2} \mathrm{~h}^{-1}$. The 398 thin layer modified membrane also showed stable desalination performance in a long-term (12 399 hours) DCMD experiment (Fig. S3 of the Supplementary Data). We attribute the observed high 400 MD performance of the thin layer modified membrane to its unique structural properties. As 401 shown previously, a thin polymer coating allows for the control of the pore size of the 402 electrospun substrate without a significant reduction in porosity, producing membranes with an 403 asymmetric structure (i.e., a dense layer on top of a highly porous substrate). The reduced pore 404 size of the membrane top layer contributes to increasing LEP, thereby enhancing surface wetting 405 resistance, while a highly porous substrate minimizes resistance to water vapor transport, 406 resulting in high water permeability. 

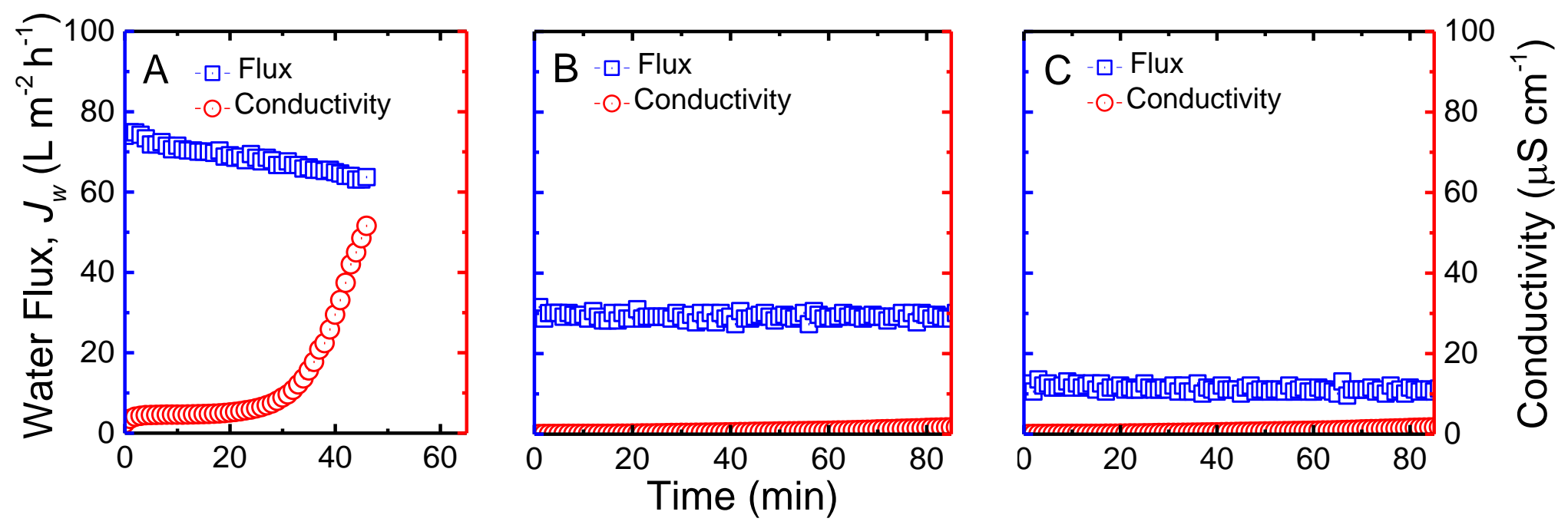

407 Fig. 6. Water flux and solution conductivity change in the permeate collector reservoir, evaluated 408 using a laboratory-scale direct contact membrane distillation (DCMD) unit. (A) PVDF-HFP 409 electrospun nanofibers (control). (B) PVDF-HFP electrospun nanofibers modified by shallow 410 infiltration of the dope solution followed by phase inversion. (C) PVDF-HFP electrospun 411 nanofibers modified by deep infiltration of the dope solution followed by phase inversion. 412 Experimental conditions were as follows: feed solution of $1 \mathrm{M} \mathrm{NaCl}$, feed and distillate 413 crossflow velocities of 15.2 and $7.6 \mathrm{~cm} / \mathrm{s}$, and feed and distillate temperatures of 60 and $20{ }^{\circ} \mathrm{C}$, 414 respectively.

\section{Conclusion}

417 We developed a method to integrate polymeric materials within a highly porous electrospun 418 mat for MD membrane fabrication. Controlling the penetration depth of the dope solution, and, 419 consequently, the thickness of the polymeric coating layer on the electrospun substrate allows for 420 tunable membrane structural parameters and performance without compromising surface 421 hydrophobicity. Proper choice for a solvent which does not damage the polymeric fibers and 422 control over the polymer loading were found to be important factors that enabled membrane 423 properties suitable for MD application. The electrospun fiber substrates coated with a controlled 424 polymeric layer thickness exhibited stable desalination performance in MD due to the enhanced 425 surface wetting resistance. The proposed coating technique is suitable for large scale electrospun 
membrane fabrication for a wide range of other applications, including size-based air and water

427 filtration systems.

\section{Acknowledgements}

430 The authors are grateful for the financial support received from the Advanced Research Projects 431 Agency-Energy (ARPA-E), U.S. Department of Energy, via Grant DE-AR0000306.

\section{References}

[1] P. Wang, T.-S. Chung, Recent advances in membrane distillation processes: Membrane development, configuration design and application exploring, Journal of Membrane Science, 474 436 (2015) 39-56.

[2] P. Wang, Y. Cui, Q. Ge, T.F. Tew, T.-S. Chung, Evaluation of hydroacid complex in the forward osmosis-membrane distillation (FO-MD) system for desalination, Journal of Membrane Science, 494 (2015) 1-7.

[3] S. Lin, N.Y. Yip, T.Y. Cath, C.O. Osuji, M. Elimelech, Hybrid pressure retarded osmosismembrane distillation system for power generation from low-grade heat: Thermodynamic analysis and energy efficiency, Environmental science \& technology, 48 (2014) 5306-5313.

443 [4] E. Shaulsky, C. Boo, S. Lin, M. Elimelech, Membrane-Based Osmotic Heat Engine with 444 Organic Solvent for Enhanced Power Generation from Low-Grade Heat, Environmental science 445 \& technology, 49 (2015) 5820-5827.

446 [5] M. Xie, L.D. Nghiem, W.E. Price, M. Elimelech, A forward osmosis-membrane distillation hybrid process for direct sewer mining: system performance and limitations, Environmental science \& technology, 47 (2013) 13486-13493.

[6] M. Xie, L.D. Nghiem, W.E. Price, M. Elimelech, Toward resource recovery from wastewater: extraction of phosphorus from digested sludge using a hybrid forward osmosis-membrane distillation process, Environmental Science \& Technology Letters, 1 (2014) 191-195.

452 [7] L.D. Tijing, Y.C. Woo, J.-S. Choi, S. Lee, S.-H. Kim, H.K. Shon, Fouling and its control in 453 membrane distillation - a review, Journal of Membrane Science, 475 (2015) 215-244.

454 [8] C. Boo, J. Lee, M. Elimelech, Engineering Surface Energy and Nanostructure of 455 Microporous Films for Expanded Membrane Distillation Applications, Environmental Science \& 456 Technology, 50 (2016) 8112-8119.

457 [9] J. Lee, C. Boo, W.-H. Ryu, A.D. Taylor, M. Elimelech, Development of Omniphobic 458 Desalination Membranes Using a Charged Electrospun Nanofiber Scaffold, ACS applied 459 materials \& interfaces, 8 (2016) 11154-11161.

460 [10] A. Alkhudhiri, N. Darwish, N. Hilal, Membrane distillation: A comprehensive review, 461 Desalination, 287 (2012) 2-18. 
462

463

464

465

466

467

468

469

470

471

472

473

474

475

476

477

478

479

480

481

482

483

484

485

486

487

488

489

490

491

492

493

494

495

496

497

498

499

500

501

[11] S. Lin, N.Y. Yip, M. Elimelech, Direct contact membrane distillation with heat recovery: Thermodynamic insights from module scale modeling, Journal of Membrane Science, 453 (2014) 498-515.

[12] M. El-Bourawi, Z. Ding, R. Ma, M. Khayet, A framework for better understanding membrane distillation separation process, J Membrane Sci, 285 (2006) 4-29.

[13] A. Alklaibi, N. Lior, Membrane-distillation desalination: status and potential, Desalination, 171 (2005) 111-131.

[14] Z.-M. Huang, Y.-Z. Zhang, M. Kotaki, S. Ramakrishna, A review on polymer nanofibers by electrospinning and their applications in nanocomposites, Composites science and technology, 63 (2003) 2223-2253.

[15] J. Doshi, D.H. Reneker, Electrospinning process and applications of electrospun fibers, in: Industry Applications Society Annual Meeting, 1993., Conference Record of the 1993 IEEE, IEEE, 1993, pp. 1698-1703.

[16] T.J. Sill, H.A. von Recum, Electrospinning: applications in drug delivery and tissue engineering, Biomaterials, 29 (2008) 1989-2006.

[17] A.F. De Faria, F.o. Perreault, E. Shaulsky, L.H. Arias Chavez, M. Elimelech, Antimicrobial Electrospun Biopolymer Nanofiber Mats Functionalized with Graphene Oxide-Silver Nanocomposites, ACS applied materials \& interfaces, 7 (2015) 12751-12759.

[18] W.-H. Ryu, H. Wilson, S. Sohn, J. Li, X. Tong, E. Shaulsky, J. Schroers, M. Elimelech, A.D. Taylor, Heterogeneous WS x/WO3 Thorn-Bush Nanofiber Electrodes for Sodium-Ion Batteries, ACS nano, 10 (2016) 3257-3266.

[19] C. Boo, J. Lee, M. Elimelech, Omniphobic Polyvinylidene Fluoride (PVDF) Membrane for Desalination of Shale Gas Produced Water by Membrane Distillation, Environmental Science \& Technology, 50 (2016) 12275-12282.

[20] Y. Liao, R. Wang, M. Tian, C. Qiu, A.G. Fane, Fabrication of polyvinylidene fluoride (PVDF) nanofiber membranes by electro-spinning for direct contact membrane distillation, Journal of Membrane Science, 425-426 (2013) 30-39.

[21] Z. Dong, S.J. Kennedy, Y. Wu, Electrospinning materials for energy-related applications and devices, Journal of Power Sources, 196 (2011) 4886-4904.

[22] P.-G. De Gennes, F. Brochard-Wyart, D. Quéré, Capillarity and wetting phenomena: drops, bubbles, pearls, waves, Springer Science \& Business Media, 2013.

[23] V.A. Ganesh, A.S. Nair, H.K. Raut, T.T.Y. Tan, C. He, S. Ramakrishna, J. Xu, Superhydrophobic fluorinated POSS-PVDF-HFP nanocomposite coating on glass by electrospinning, Journal of Materials Chemistry, 22 (2012) 18479-18485.

[24] B.S. Lalia, E. Guillen-Burrieza, H.A. Arafat, R. Hashaikeh, Fabrication and characterization of polyvinylidenefluoride-co-hexafluoropropylene (PVDF-HFP) electrospun membranes for direct contact membrane distillation, Journal of Membrane Science, 428 (2013) 104-115.

[25] S. Kaur, R. Barhate, S. Sundarrajan, T. Matsuura, S. Ramakrishna, Hot pressing of electrospun membrane composite and its influence on separation performance on thin film composite nanofiltration membrane, Desalination, 279 (2011) 201-209. 
[26] M. Khayet, T. Matsuura, MD Membrane Characterization, (2011) 189-225.

503 [27] D. Li, Y. Xia, Electrospinning of nanofibers: reinventing the wheel?, Advanced materials, $504 \quad 16$ (2004) 1151-1170.

505 [28] J.R.K. Sung Won Choi, Young Rack Ahn, Seong Mu Jo, and, E.J. Cairns, Characterization 506 of Electrospun PVdF Fiber-Based Polymer Electrolytes, Chem. Mater., 19 ( 2007) 104 - 115.

507 [29] E.N. Dalal, Calculation of solid surface tensions, Langmuir, 3 (1987) 1009-1015.

508 [30] F.A. Dullien, Porous media: fluid transport and pore structure, Academic press, 2012.

509 [31] F.C. Krebs, Fabrication and processing of polymer solar cells: a review of printing and 510 coating techniques, Solar Energy Materials and Solar Cells, 93 (2009) 394-412.

511 [32] M. Buonomenna, P. Macchi, M. Davoli, E. Drioli, Poly (vinylidene fluoride) membranes by 512 phase inversion: the role the casting and coagulation conditions play in their morphology, 513 crystalline structure and properties, European Polymer Journal, 43 (2007) 1557-1572.

514 [33] C.M. Hansen, Hansen solubility parameters: a user's handbook, CRC press, 2007.

515 [34] S. Nejati, C. Boo, C.O. Osuji, M. Elimelech, Engineering flat sheet microporous PVDF 516 films for membrane distillation, Journal of Membrane Science, 492 (2015) 355-363.

517 [35] D. Blundell, A. Keller, A. Kovacs, A new self- nucleation phenomenon and its application 518 to the growing of polymer crystals from solution, Journal of Polymer Science Part B: Polymer 519 Letters, 4 (1966) 481-486.

520 [36] A. Ahmad, N. Ideris, B. Ooi, S. Low, A. Ismail, Morphology and polymorph study of a polyvinylidene fluoride (PVDF) membrane for protein binding: Effect of the dissolving temperature, Desalination, 278 (2011) 318-324.

[37] M. Tomaszewska, Preparation and properties of flat-sheet membranes from poly (vinylidene fluoride) for membrane distillation, Desalination, 104 (1996) 1-11.

[38] P. Sukitpaneenit, T.-S. Chung, Molecular elucidation of morphology and mechanical properties of PVDF hollow fiber membranes from aspects of phase inversion, crystallization and rheology, Journal of Membrane Science, 340 (2009) 192-205.

[39] W. Pu, X. He, L. Wang, C. Jiang, C. Wan, Preparation of PVDF-HFP microporous membrane for Li-ion batteries by phase inversion, Journal of membrane science, 272 (2006) 1114.

[40] C.-Y. Kuo, H.-N. Lin, H.-A. Tsai, D.-M. Wang, J.-Y. Lai, Fabrication of a high hydrophobic PVDF membrane via nonsolvent induced phase separation, Desalination, 233 (2008) 40-47.

[41] L.-Y. Yu, Z.-L. Xu, H.-M. Shen, H. Yang, Preparation and characterization of PVDF-SiO 2 composite hollow fiber UF membrane by sol-gel method, Journal of Membrane Science, 337 (2009) 257-265.

[42] J.F. Li, Z.L. Xu, H. Yang, C.P. Feng, J.H. Shi, Hydrophilic microporous PES membranes prepared by PES/PEG/DMAc casting solutions, Journal of applied polymer science, 107 (2008) 4100-4108. 
540 [43] C. Yao, X. Li, K. Neoh, Z. Shi, E. Kang, Antibacterial activities of surface modified 541 electrospun poly (vinylidene fluoride-co-hexafluoropropylene)(PVDF-HFP) fibrous membranes, 542 Applied Surface Science, 255 (2009) 3854-3858.

543 [44] A. Tuteja, W. Choi, M. Ma, J.M. Mabry, S.A. Mazzella, G.C. Rutledge, G.H. McKinley,

544 R.E. Cohen, Designing superoleophobic surfaces, Science, 318 (2007) 1618-1622.

545 [45] M. Miwa, A. Nakajima, A. Fujishima, K. Hashimoto, T. Watanabe, Effects of the surface 546 roughness on sliding angles of water droplets on superhydrophobic surfaces, Langmuir, 16 (2000) $547 \quad 5754-5760$.

548 [46] A. Nakajima, A. Fujishima, K. Hashimoto, T. Watanabe, Preparation of transparent 549 superhydrophobic boehmite and silica films by sublimation of aluminum acetylacetonate, 550 Advanced Materials, 11 (1999) 1365-1368.

551 [47] S. Srinivasan, G.H. McKinley, R.E. Cohen, Assessing the accuracy of contact angle 552 measurements for sessile drops on liquid-repellent surfaces, Langmuir, 27 (2011) 13582-13589.

553 [48] K. Smolders, A. Franken, Terminology for membrane distillation, Desalination, 72 (1989) $554249-262$.

555 [49] A. Wessel, On using the effective contact angle and the water drop penetration time for 556 classification of water repellency in dune soils, Earth Surface Processes and Landforms, 13 557 (1988) 555-561.

558 [50] M.C. Garcia-Payo, M.A. Izquierdo-Gil, C. Fernandez-Pineda, Wetting Study of 559 Hydrophobic Membranes via Liquid Entry Pressure Measurements with Aqueous Alcohol 560 Solutions, J Colloid Interface Sci, 230 (2000) 420-431.

561 [51] D. Murakami, H. Jinnai, A. Takahara, Wetting transition from the Cassie-Baxter state to the 562 Wenzel state on textured polymer surfaces, Langmuir, 30 (2014) 2061-2067.

563 [52] C. Huh, S. Mason, Effects of surface roughness on wetting (theoretical), Journal of colloid 564 and interface science, 60 (1977) 11-38.

565 [53] M. Gryta, Effectiveness of water desalination by membrane distillation process, Membranes, 5662 (2012) 415-429. 